\title{
DEFICIÊNCIA DE VITAMINA B12 NO PÓS-OPERATÓRIO DE CIRURGIA BARIÁTRICA: UMA REVISÃO DE LITERATURA
}

\author{
VITAMIN B12 DEFICIENCY AFTER BARIATRIC SURGERY: A LITERATURE \\ REVIEW \\ Sara Ariana de Sousa Dourado, Lucélia Oliveira Paula
}

Centro Universitário de Saúde, Ciências Humanas e Tecnológicas do Piauí - UNINOVAFAPI

\begin{abstract}
This article discusses vitamin B12 deficiency and its reflexes in the health of people undergoing bariatric surgery. This is an exploratory descriptive study of the type literature review in which a search was conducted for articles from journals indexed in the Pubmed/MEDLINE and Scielo databases, using the descriptors bariatric surgery, obesity, postoperative care, malnutrition and vitamin $B 12$ in order to gather information about this deficiency and its reflexes on the health of this population. The inclusion criteria were work on the theme from 2005 to 2016. With this, 35 articles were selected. The findings confirmed the existence of malnutrition of vitamin B12 in people who underwent this type of surgery and explained the importance of its longterm supplementation for its prevention and/or treatment, and although there are no specific recommendations for this population yet, it has been a preventive clinical practice to administer $1000 \mu \mathrm{g}$ parenteral in the preoperative period and in the postoperative period may be oral (2000 mcg/120 days) or intramuscular (1000 mcg injections). Therefore, this deficiency in bariatric patients is evidenced in the current literature that elucidates their health reflexes, demonstrating the importance of their supplementation before and after surgery, but there is a need for more specific studies that help in the creation of precise protocols.
\end{abstract}

Key words: Bariatric Surgery, Obesity, Postoperative Care, Malnutrition, Vitamin B 12
Resumo

Este artigo aborda sobre a deficiência de vitamina B12 e seus reflexos na saúde de pessoas submetidas à cirurgia bariátrica. Trata-se de um estudo descritivo exploratório do tipo revisão de literatura em que foi realizada uma pesquisa por artigos de periódicos indexados nas bases de dados Pubmed/MEDLINE e Scielo, utilizando os descritores cirurgia bariátrica, obesidade, cuidados pós-operatórios, deficiências nutricionais e vitamina b12 com o objetivo de reunir informações sobre essa deficiência e seus reflexos na saúde dessa população. Os critérios de inclusão foram trabalhos com a temática do período de 2005 à 2016. Com isso, foram selecionados 35 artigos. Os achados confirmaram a existência de deficiência nutricional de vitamina B12 em pessoas que passaram por esse tipo de cirurgia e explicitaram a importância da sua suplementação a longo tempo para sua prevenção e/ou tratamento e embora ainda não existam recomendações específicas para essa população, tem sido uma prática clínica preventiva administrar $1.000 \mu \mathrm{g}$ parenteral no período pré-operatório e no pós-operatório o tratamento pode ser por via oral $(2.000 \mathrm{mcg} / 120$ dias) ou intramuscular (injeções de $1.000 \mathrm{mcg}$ ). Portanto, essa deficiência em pacientes bariátricos é evidenciada na literatura vigente que elucida seus reflexos na saúde, demonstrando a importância de sua suplementação antes e após a cirurgia, porém há necessidade de mais estudos específicos que auxiliem na criação de protocolos precisos.

Palavras chave: cirurgia bariátrica, obesidade, cuidados pós-operatórios, deficiências nutricionais, vitamina b12 


\section{Introdução}

A obesidade é um agravo multifatorial originado, na maioria dos casos, pelo desbalanço energético, quando o indivíduo consome mais energia do que gasta resultando em ganho de peso ${ }^{1}$, na qual ocorre um acúmulo excessivo de tecido adiposo que pode ou não estar associado a outras enfermidades como: hipertensão arterial sistêmica, diabete melito tipo 2, apneia obstrutiva do sono e dislipidemias ${ }^{2}$.

Sua prevalência vem aumentando significativamente em diversas populações do mundo, incluindo o Brasil ${ }^{3}$. Dados do VIGITEL $2015^{4}$ demonstraram esse aumento na população adulta do Brasil de 11,8\% (porcentagem da prevalência de obesidade em 54369 entrevistados) para 18,9\% (porcentagem da prevalência de obesidade em 54174 entrevistados), no período de 2006 a 2015 respectivamente, representando um crescimento médio de $0,77 \%$ ao ano.

A orientação dietética mais a programação de atividade física e o uso de fármacos antiobesidade são os pilares principais do tratamento da obesidade ${ }^{5}$. Entretanto, essa terapia clínica tem sucesso limitado a curto prazo e quase inexistente a longo prazo ${ }^{5}$. Com isso, o tratamento cirúrgico é indicado e tem se mostrado mais eficaz a longo prazo, devendo basear-se numa análise abrangente de múltiplos aspectos clínicos incluindo uma avaliação desses pacientes no pré e pós-operatório por uma equipe multidisciplinar ${ }^{5-10}$.

As técnicas cirúrgicas podem ser divididas em procedimentos restritivos (diminuem a entrada de alimento e promovem uma rápida sensação de saciedade após refeições), procedimentos disabsortivos (reduzem a absorção das calorias, proteínas e outros nutrientes) e procedimentos mistos (é a combinação de ambas as técnicas) ${ }^{11,12}$

Contudo, essas cirurgias sabidamente têm como consequência, além da perda de peso, uma deficiência de nutrientes. Dentre estes, está a vitamina B12 que é uma das deficiências mais frequentes no pós-cirúrgico podendo ocorrer por diversos fatores, como hipocloridria gástrica, má absorção ileal devido produção inadequada do fator intrínseco no estômago, ressecção ileal e intolerância alimentar a alimentos fontes ${ }^{6,13-21}$.

A vitamina B12 é essencial em numerosas reações bioquímicas, como a transferência de grupos metílicos e reações de metilação, importantes na síntese e metabolismo de neurotransmissores e fosfolipídeos do sistema nervoso central. Além de participar da síntese de ácidos nucléicos e no metabolismo de ácidos graxos e aminoácidos que se incorporam ao ciclo dos ácidos tricarboxílicos na mitocôndria ${ }^{22}$. Ao mesmo tempo, assim como o ferro e o ácido fólico, ela também é essencial para o desenvolvimento e divisão celular, bem como para a produção de hemácias e de material genético. Não obstante, sua deficiência pode causar anemia perniciosa, sintomas neurológicos e fraqueza ${ }^{23}$.

Dessa forma, considerando que o tratamento da obesidade em muitos casos requer a realização de procedimentos cirúrgicos que apresentam no seu pós-operatório intercorrências como deficiências nutricionais, dentre elas a carência de vitamina B12, esta revisão tem por objetivo reunir informações da literatura científica sobre esta deficiência de vitamina B12 em pacientes pós-cirurgia bariátrica analisando os reflexos dessa carência na saúde das pessoas que se submeteram a esse tratamento cirúrgico.

\section{Metodologia}

Trata-se de um estudo descritivo exploratório do tipo revisão de literatura integrativa. Para o presente estudo foi realizada uma pesquisa, no período de março a outubro de 2016, por artigos de periódicos indexados nas bases de dados Pubmed/MEDLINE e Scielo. Para as buscas foram utilizados os seguintes descritores em ciências e saúde (DeCS) e seus respectivos correspondentes em inglês (Medical Subject Headings - MESH), aplicados individualmente ou combinados: cirurgia bariátrica, obesidade, cuidados pós-operatórios, deficiências nutricionais, vitamina B12. Os critérios de inclusão foram publicações originais e do tipo revisão que abordassem a temática durante o período de 2005 a 2016 nos idiomas português, inglês e espanhol, sendo excluídos aqueles que não atendessem a esses critérios e os que não foram realizados em humanos.

Após uma análise inicial com base nos títulos dos manuscritos e posterior leitura dos mesmos, 35 foram utilizados para a presente revisão. Desses artigos utilizados, 13 foram encontrados em ambos os indexadores. Após seleção criteriosa, foi observado que apenas 11 documentos se referiram à temática deficiência de vitamina B12 no pós-operatório de cirurgia bariátrica proposta por este estudo. Com base nisso, as discussões foram feitas de acordo com os resultados e explicações para tais achados nos 
trabalhos encontrados (Figura 1).

Figura 1. Fluxograma_Processo de seleção dos artigos nas bibliotecas virtuais Scielo e PubMed/MEDLINE.

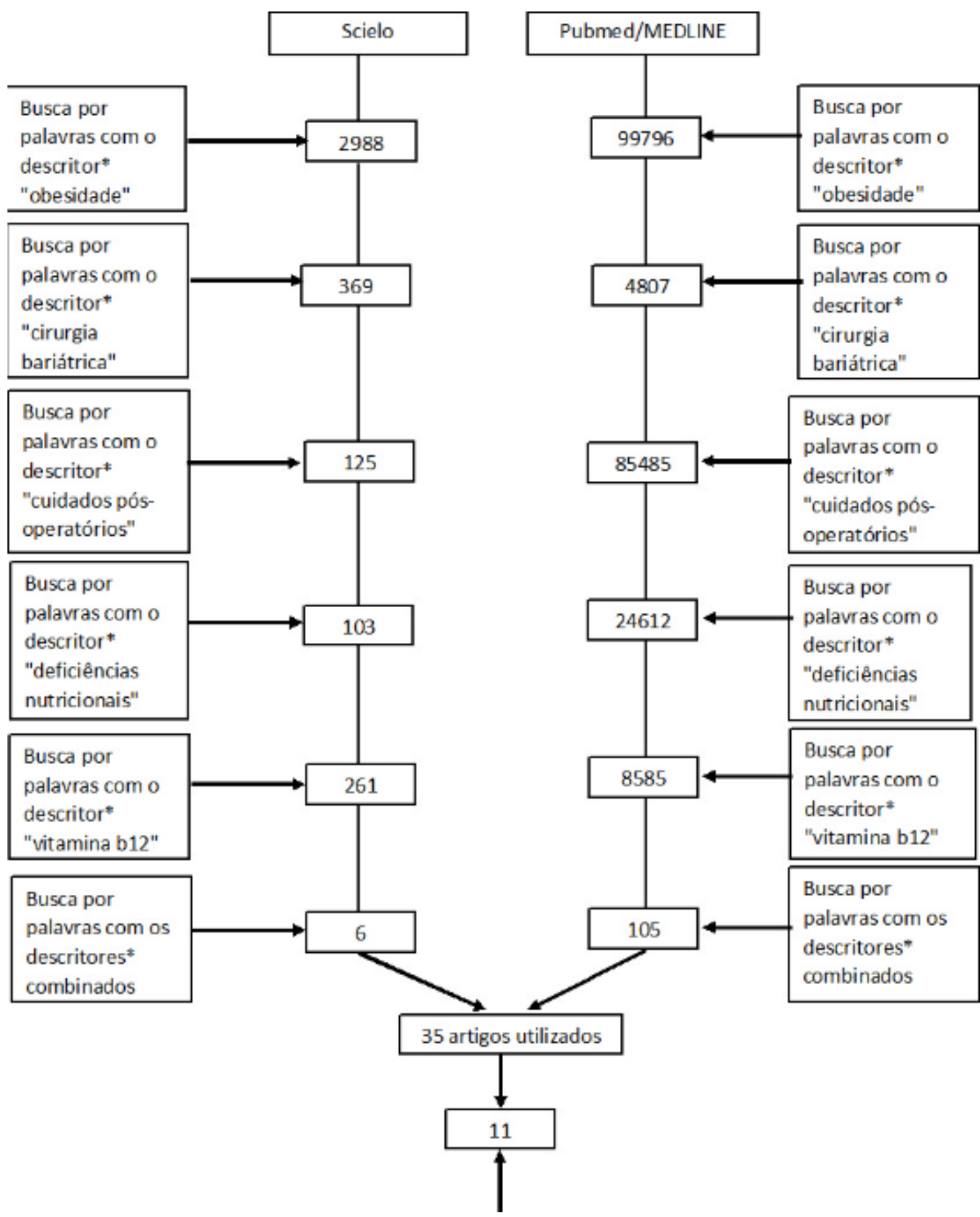

\section{Resultados e Discussões}

Foram encontrados 4807 (Pubmed) e 369 (Scielo) artigos relacionados à cirurgia bariátrica, 99796 (Pubmed) e 2988 (Scielo) artigos relacionados à obesidade, 85485 (Pubmed) e 125 (Scielo) artigos relacionados a cuidados pósoperatórios, 24612 (Pubmed) e 103 (Scielo) artigos relacionados a deficiências nutricionais e
8585 (Pubmed) e 261 (Scielo) sobre vitamina B12. Para otimizar a pesquisa foram procuradas nas bases de dados as palavras-chave combinadas e como resultado foram encontrados 105 (Pubmed) e 6 (Scielo) artigos. Acertando-se os métodos de busca, 11 artigos científicos foram selecionados para compor este estudo. A Tabela 1 descreve as informações gerais sobre eles. 
Tabela 1. Descrição dos artigos referente à revista, ano de publicação, autoria, local, natureza e objetivos do estudo.

\begin{tabular}{|c|c|c|c|c|}
\hline $\begin{array}{l}\text { Revista (Ano } \\
\text { de } \\
\text { publicação) }\end{array}$ & Autoria & $\begin{array}{l}\text { Local de } \\
\text { realização }\end{array}$ & $\begin{array}{l}\text { Tipo de } \\
\text { estudo }\end{array}$ & Objetivos do estudo \\
\hline $\begin{array}{l}\text { Arq Bras } \\
\text { Endocrinol } \\
\text { Metab } \\
\text { (2006) }\end{array}$ & $\begin{array}{l}\text { Alves, } \\
\text { Gonçalves, } \\
\text { Cordeiro, } \\
\text { Lauria, Ramos }\end{array}$ & $\begin{array}{l}\text { Belo } \\
\text { Horizonte, MG }\end{array}$ & $\begin{array}{l}\text { Estudo de } \\
\text { dois casos e } \\
\text { Revisão de } \\
\text { literatura }\end{array}$ & $\begin{array}{l}\text { Alertar sobre a importância da vigilância } \\
\text { nutricional após a cirurgia bariátrica. }\end{array}$ \\
\hline $\begin{array}{l}\text { Nutrition } \\
(2010)\end{array}$ & $\begin{array}{l}\text { Shankar, } \\
\text { Boylan, Sriram }\end{array}$ & $\begin{array}{l}\text { Estados } \\
\text { Unidos }\end{array}$ & $\begin{array}{l}\text { Revisão } \\
\text { sistemática }\end{array}$ & $\begin{array}{l}\text { Discorrer sobre as deficiências de } \\
\text { micronutrientes e seus sintomas após } \\
\text { cirurgia bariátrica. }\end{array}$ \\
\hline $\begin{array}{l}\text { Rev. Assoc. } \\
\text { Med. Bras } \\
(2011)\end{array}$ & $\begin{array}{l}\text { Bordalo, } \\
\text { Teixeira, } \\
\text { Bressan, } \\
\text { Mourão }\end{array}$ & Viçosa, MG & $\begin{array}{l}\text { Revisão } \\
\text { sistemática }\end{array}$ & $\begin{array}{l}\text { Auxiliar a melhor seleção de nutrientes de } \\
\text { forma a garantir uma reposição adequada } \\
\text { dos nutrientes em pacientes submetidos à } \\
\text { cirurgia bariátrica. }\end{array}$ \\
\hline $\begin{array}{l}\text { Arq Bras Cir } \\
\text { Dig (2012) }\end{array}$ & $\begin{array}{l}\text { Carvalho, } \\
\text { Loscalzo, } \\
\text { Freitas, Jordão, } \\
\text { Friano }\end{array}$ & Campinas, SP & $\begin{array}{l}\text { Estudo } \\
\text { retrospectivo } \\
\text { observacional }\end{array}$ & $\begin{array}{l}\text { Verificar a incidência da deficiência de } \\
\text { vitamina B12 e comparar dados } \\
\text { antropométricos e bioquímicos do pré e } \\
\text { pós-operatório (seis meses), em pacientes } \\
\text { submetidos à cirurgia bariátrica Fobi- } \\
\text { Capella (Y de Roux). }\end{array}$ \\
\hline $\begin{array}{l}\text { BioMedCent } \\
\text { ral Surgery } \\
(2012)\end{array}$ & $\begin{array}{l}\text { Pech, Meyer, } \\
\text { Lippert, } \\
\text { Manger, Stroh }\end{array}$ & $\begin{array}{l}\text { Gera, } \\
\text { Alemanha }\end{array}$ & $\begin{array}{l}\text { Estudo } \\
\text { prospectivo } \\
\text { observacional }\end{array}$ & $\begin{array}{l}\text { Investigar os resultados e as deficiências } \\
\text { nutricionais de pacientes após gastrectomia } \\
\text { vertical durante um acompanhamento } \\
\text { médio de dois anos. }\end{array}$ \\
\hline $\begin{array}{l}\text { SpringerPlus } \\
\text { Journal } \\
\text { (2013) }\end{array}$ & $\begin{array}{l}\text { Eltweri, } \\
\text { Bowrey, } \\
\text { Sutton, } \\
\text { Graham, } \\
\text { Williams }\end{array}$ & $\begin{array}{l}\text { Estados } \\
\text { Unidos da } \\
\text { América }\end{array}$ & $\begin{array}{l}\text { Estudo } \\
\text { retrospectivo }\end{array}$ & $\begin{array}{l}\text { Identificar qualquer deficiência de vitamina } \\
\text { B12 e destacar a necessidade de } \\
\text { monitorização pós-laparoscópica de } \\
\text { gastroenterologia com vitamina B12. }\end{array}$ \\
\hline $\begin{array}{l}\text { Medicine, } \\
\text { Journal } \\
(2014)\end{array}$ & $\begin{array}{l}\text { Dogan, Aarts, } \\
\text { Koehestanie, } \\
\text { Betzel, Ploeger, } \\
\text { Boer, et al. }\end{array}$ & Países Baixos & $\begin{array}{l}\text { Ensaio clínico } \\
\text { triplo-cego, } \\
\text { randomizado }\end{array}$ & $\begin{array}{l}\text { Avaliar a eficácia e a segurança do } \\
\text { multivitamínico WLS Forte em comparação } \\
\text { com o padrão MVS (comprimidos } \\
\text { comercialmente disponíveis) após bypass } \\
\text { gástrico Y de Roux em um ensaio } \\
\text { controlado randomizado triplo-cego. }\end{array}$ \\
\hline $\begin{array}{l}\text { Arq Bras Cir } \\
\text { Dig (2014) }\end{array}$ & $\begin{array}{l}\text { Leiro, } \\
\text { Melendez- } \\
\text { Araújo }\end{array}$ & Brasília, DF & $\begin{array}{l}\text { Estudo } \\
\text { transversal } \\
\text { descritivo }\end{array}$ & $\begin{array}{l}\text { Determinar as quantidades de ferro, cálcio, } \\
\text { vitamina D e vitamina B12 dietéticas } \\
\text { ingeridas por pacientes atendidos em um } \\
\text { hospital público após um ano de bypass } \\
\text { gástrico, e comparar com as } \\
\text { recomendações da Recommended Dietary } \\
\text { Allowances. }\end{array}$ \\
\hline $\begin{array}{l}\text { Arq Bras Cir } \\
\text { Dig (2015) }\end{array}$ & $\begin{array}{l}\text { Ramos, Magno, } \\
\text { Cohen, Rosado, } \\
\text { Carneiro }\end{array}$ & $\begin{array}{l}\text { Rio de Janeiro, } \\
\text { RJ }\end{array}$ & $\begin{array}{l}\text { Estudo } \\
\text { retrospectivo } \\
\text { observacional }\end{array}$ & $\begin{array}{l}\text { Avaliar a evolução da perda ponderal e a } \\
\text { presença de anemias carenciais em } \\
\text { pacientes submetidos ao bypass gástrico } \\
\text { em Y-de-Roux em uso de suplementação de } \\
\text { vitaminas e minerais. }\end{array}$ \\
\hline $\begin{array}{l}\text { Arq Bras Cir } \\
\text { Dig (2015) }\end{array}$ & $\begin{array}{l}\text { Santos, Burgos, } \\
\text { Lemos, Cabral }\end{array}$ & Recife, PE & $\begin{array}{l}\text { Estudo } \\
\text { retrospectivo } \\
\text { não } \\
\text { concorrente } \\
\text { do tipo série } \\
\text { de casos }\end{array}$ & $\begin{array}{l}\text { Avaliar a evolução clínico-nutricional } \\
\text { durante o primeiro ano de mulheres obesas } \\
\text { submetidas ao bypass gástrico em Y-de- } \\
\text { Roux. }\end{array}$ \\
\hline $\begin{array}{l}\text { Arq Bras Cir } \\
\text { Dig (2015) }\end{array}$ & $\begin{array}{l}\text { Silveira-Júnior, } \\
\text { Albuquerque, } \\
\text { Nascimento, } \\
\text { Rosa, Hygidio, } \\
\text { Zapelini }\end{array}$ & Tubarão, SC & $\begin{array}{l}\text { Estudo de } \\
\text { coorte, } \\
\text { prospectivo, } \\
\text { analítico e } \\
\text { descritivo }\end{array}$ & $\begin{array}{l}\text { Identificar alterações nutricionais em } \\
\text { pacientes submetidos à cirurgia bariátrica e } \\
\text { correlacioná-las com perda de peso, } \\
\text { controle de comorbidades e qualidade de } \\
\text { vida. }\end{array}$ \\
\hline
\end{tabular}




\section{Conclusão}

A primeira terapêutica para a obesidade inclui tratamentos dietético, psicológico, fisioterápico e exercícios físicos, além do uso de medicamentos anorexígenos ou disabsortivos ${ }^{24}$. Porém, se esta não apresentar resultados significativos a longo prazo, a operação bariátrica se torna a ferramenta mais eficaz e segura no controle e tratamento da obesidade mórbida, proporcionando uma redução de peso sustentada de $30-40 \%$ do peso inicial, além da melhora da qualidade de vida e da maioria das co-morbidades associadas, como diabetes tipo 2, hipertensão arterial sistêmica e disfunção cardiovascular $^{25,26}$.

Os critérios de indicação da cirurgia bariátrica incluem portadores de obesidade de grandes proporções, de duração superior a dois anos, com Índice de Massa Corporal (IMC) maior que $40 \mathrm{~kg} / \mathrm{m}^{2}$, resistentes a tratamentos conservadores (dietas, exercícios, medicamentos, psicoterapia), sendo ainda indicados para obesos com IMC superior a $35 \mathrm{~kg} / \mathrm{m}^{2}$ portadores de comorbidades como diabetes melito, dislipidemias, doenças cardiovasculares, asma grave não controlada, apnéia do sono, artropatias, hérnias discais, refluxos gastroesofágicos com indicação cirúrgica, esteatose hepática, infertilidade masculina e feminina, dentre outras que tenham sua situação clínica agravada pela obesidade ${ }^{27}$.

$E$ as principais contraindicações são a ausência de tratamento clínico ou de gestão, a existência de doenças psiquiátricas ativas não estabilizadas (depressão e transtornos psicóticos), dependências em álcool e/ou drogas, doenças que ameaçam a vida no curto prazo, e aqueles incapazes de cuidar de si mesmos, sem família ou apoio social ${ }^{27}$.

Com relação às complicações pósoperatórias, o paciente pode desenvolver, em longo prazo, deficiências de alguns macros e micronutrientes. Os possíveis mecanismos são: ingestão nutricional deficiente, má absorção decorrente da técnica cirúrgica, pobre aderência à reposição de polivitamínicos e a presença de sintomas gastrintestinais, além das intolerâncias alimentares que também podem ocorrer ${ }^{13,16,25,28-}$ 31.

Essas complicações podem variar de acordo com a técnica cirúrgica submetida. Nas técnicas restritivas (balão intragástrico, Banda Gástrica Ajustável - BGA e Gastroplastia Vertical com Bandagem - GVB, também conhecida como cirurgia de Mason) ocorre restrição do conteúdo gástrico, provocando saciedade precoce com pequena quantidade de alimentos ingerida. Já as técnicas consideradas mistas (derivação gástrica em Y-de-Roux, cirurgia de Scopinaro/Derivação Biliopancreática - DBP com gastrectomia distal e Derivação Biliopancreática com gastrectomia vertical e preservação do piloro - DBP/DS) apresentam uma parcela restritiva e evitam ou diminuem a absorção de nutrientes por meio de uma derivação no intestino delgado, diminuindo sua área absortiva ${ }^{12,32,33}$.

Devido a existência dessas complicações, há uma necessidade de acompanhamento nutricional periódico a longo prazo que garanta uma alimentação adequada para um resultado promissor do pós-operatório ${ }^{34}$. Vale ressaltar que possíveis deficiências no pré-operatório também devem ser investigadas a fim de serem corrigidas precocemente, possibilitando que déficits desenvolvidos antes da operação não sejam confundidos com complicações pósoperatórias ${ }^{13}$.

Dentre os distúrbios nutricionais após a cirurgia bariátrica, os mais freqüentes são a desnutrição protéica, as deficiências de ferro e zinco e as deficiências vitamínicas, como a vitamina B12, que parecem ser mais significativas após procedimentos disabsortivos, não descartando a ocorrência nos restritivos ${ }^{16,19,25}$.

As causas da deficiência de vitamina B12 em pós-operados de cirurgia bariátrica são a ingestão limitada de proteínas de origem animal, redução das secreções gástricas que prejudicam a clivagem da vitamina a partir da proteína e diminuição na produção de fator intrínseco pelo estômago, evidenciando que mesmo com a ingestão de alimentos fonte suficiente, a absorção desse micronutriente provavelmente encontra-se muito debilitada ${ }^{13,18}$.

Contudo, podem ocorrer também intolerâncias alimentares no pós-operatório ${ }^{13}$. Alguns autores (Silva et al., Carvalho et al., Costa et al., Eltweri et al., Traina et al., Schweiger et al., Moreira et al.) 12,16,19,21,36-38 relatam em seus estudos sobre pacientes submetidos à cirurgia bariátrica que a carne vermelha foi o principal alimento responsável por essas intolerâncias, podendo, assim, explicar a deficiência da cobalamina, já que esta é disponibilizada em alimentos de origem animal e sua absorção no íleo depende da presença de fator intrínseco liberado pelas células parietais do estômago ${ }^{16,35}$.

Essa intolerância à carne pode ocorrer devido à significativa ressecção gástrica proporcionada pela operação, tendo consequentemente alteração na produção de 
pepsina, responsável principal pela digestão das proteínas $^{38}$.

Estudos 14,16-18,20,21, como os de Santos et al. (retrospectivo não concorrente do tipo série de casos com 61 mulheres que analisou variáveis antropométricas, perfil lipídico, uso de polivitamínico-mineral, prática de exercício físico e presença de sintomas associados), Carvalho et al. (análise retrospectiva e descritiva de 91 prontuários de pacientes submetidos à operação que avaliou redução de peso, comorbidades, taxas sanguíneas e vitamina B12 antes e após - 6 meses o procedimento), Dogan et al. (estudo duplo-cego, randomizado de 12 meses que comparou multivitamínico WLS forte com um padrão em 148 pacientes, 74 em cada grupo, após bypass gástrico em $Y$ de Roux), Ramos et al. (análise retrospectiva de 137 pacientes suplementados com polivitaminas e minerais, incluindo dados antropométricos e laboratoriais nos períodos pré-operatório e 12, 24, 36 e 48 meses de pós-operatório), Silveira-Júnior et al. (estudo de coorte prospectivo, analítico e descritivo de dados coletados no pré-operatório e aos três e seis meses pós-operatórios de 59 pacientes submetidos à cirurgia bariátrica, quantificando aspectos nutricionais pela ferramenta Bariatric Analysis and Reporting Outcomes System - BAROS) e Eltweri et al. (estudo retrospectivo de 41 pacientes submetidos a gastrectomia vertical e após o procedimento, suplementados com multivitamínico WS forte, ferro e cálcio com vitamina $D$ e submetidos a exames laboratoriais que analisaram valores de vitamina B12, selênio, folato sérico, ferritina, ferro, zinco, cobre e magnésio), confirmam essa baixa nos níveis séricos de cobalamina em pacientes póscirúrgicos, inclusive naqueles em que houve um reganho de peso ${ }^{39}$.

Já no estudo ${ }^{13}$ transversal descritivo de Leiro e Melendez-Araújo com 36 mulheres após um ano de bypass gástrico contendo dados sociodemográficos, antropométricos e alimentares (Questionário de Frequência Alimentar), comparou valores da ingestão de ferro, cálcio, vitamina D e B12 com as Recommended Dietary Allowances (RDA), assim como a correlação do tempo de pós-operatório com o consumo desses micronutrientes. Os achados desse estudo foram a inadequação na ingestão de ferro, cálcio e vitamina $D$, mas diferentes de vitamina B12 que atingiu 104,2\% de adequação, sugerindo adaptação da alimentação dos pacientes ao longo do tempo. Contudo, muitas dificuldades diagnósticas laboratoriais existem e ainda não se estabeleceu consenso para um teste que fosse considerado padrão-ouro para o diagnóstico dessa deficiência ${ }^{16}$.

Essa deficiência em pacientes pós-cirúrgicos pode apresentar, apesar de raros, sintomas diversos como cãimbras, parestesias, dor, anemia megaloblástica, anemia perniciosa, e macrocítica, deficiência de ácido fólico, neuropatia periférica e sintomas psiquiátricos, especialmente transtornos depressivos, além dos mesmos poderem apresentar leucopenia, glossite e níveis elevados de homocisteína ${ }^{14,35,40}$.

Nos casos mais graves, encefalopatia aguda de Wernicke secundária a deficiência de tiamina e vitamina B12 pode, independentemente da instituição do tratamento, induzir dano permanente ao paciente ${ }^{31}$.

Devido aos múltiplos fatores que essa deficiência pode causar, a suplementação se torna indispensável como forma de prevenção e/ou tratamento ${ }^{13,17,18}$. O estudo ${ }^{15}$ prospectivo observacional, de Pech e colaboradores, com 100 pacientes dois anos após gastrectomia vertical que avaliou nesse período os aspectos gerais da operação mais os resultados laboratoriais (ferro, zinco, selênio, vitamina B12, ácido fólico, cálcio, hormônio da paratireóide, hemoglobina, albumina e fosfatase alcalina), demonstrou que sem suplementação, teria ocorrido deficiência de vitamina B12, pois seus níveis variaram durante a suplementação mensal de $1000 \mathrm{mg}$ da vitamina.

As recomendações para ingestão de macro e micronutrientes mais utilizadas atualmente são as Recommended Dietary Allowances (RDA). Porém, as recomendações são aplicáveis apenas para populações sadias, não existindo ainda recomendações específicas para esse tipo de população. Concomitante a isso, os estoques corporais de micronutrientes diminuem com o passar do tempo, necessitando-se de um monitoramento periódico após a operação a partir de exames físicos e bioquímicos ${ }^{13}$.

Dogan et al., em um estudo ${ }^{17}$ duplo-cego e randomizado, avaliaram e compararam a suplementação vitamínica com multivitamínico padrão (de acordo com as RDA - 2,5mcg de vitamina B12) e WLS Forte (quantidade de vitamina B12 140 vezes maior que a das $R D A$, equivalente a $350 \mathrm{mcg}$ ) por 12 meses em pacientes submetidos a cirurgia gástrica de bypass em $Y$-de-Roux, e concluíram que níveis séricos de vitamina B12 diminuíram no grupo com multivitamínico padrão e aumentaram significativamente no grupo WLS Forte demonstrando que um suplemento 
multivitamínico específico para esse tipo de paciente tem vantagens sobre a suplementação padrão.

No entanto, as formas de apresentação das suplementações são importantes, pois deve-se levar em consideração $\mathrm{o} \mathrm{pH}$ gastrointestinal necessário para a solubilização do nutriente; a forma de apresentação do suplemento (solução aquosa, cápsula, pó); as enzimas presentes no trato gastrointestinal; a integridade intestinal e superfície de absorção; a via de administração (oral, intramuscular ou endovenosa) e a quantidade e tipo de micronutriente presente nas formas de administração. Além disso, o tipo de procedimento cirúrgico realizado também influencia nos cuidados com a escolha do suplemento nutricional ${ }^{6}$.

Atualmente, a suplementação de B12 está disponível no mercado também na forma de spray para ser aplicado na forma sublingual. Esse tipo de suplementação é apresentado na forma de nano partículas para aumentar a absorção e a biodisponibilidade da vitamina. Embora a deficiência de vitamina B12 ocorra apenas após alguns meses de cirurgia bariátrica, tem sido uma boa prática clínica preventiva administrar 1.000 $\mu \mathrm{g}$ de vitamina B12 de forma parenteral durante o período pré-operatório ${ }^{40}$ e no pós-operatório ${ }^{35}$ o tratamento pode ser por via oral (doses diárias de 2.000 microgramas - mcg por 120 dias) ou intramuscular (injeções de 1.000 mcg 3 vezes por semana por um período de duas semanas, seguidas de uma injeção mensal por mais três meses).

A deficiência de vitamina B12 em pessoas que se submeteram à cirurgia bariátrica é evidenciada na literatura vigente, porém observa-se uma necessidade de mais estudos específicos que tratem sobre essa deficiência em pós-operatório de cirurgia bariátrica, especialmente que comparem seus níveis com os encontrados no período pré-operatório, para que possam ser feitos protocolos mais precisos de suplementação de cobalamina para esse tipo de população, pois sua carência acarreta algumas intercorrências, como dor e sintomas físicos, além de anemias e nos casos mais graves, problemas que acometem o sistema nervoso, influenciando na qualidade de vida e de saúde dos pacientes.

\section{Referências}

1. Brasil. Ministério da Saúde, Secretaria de Atenção à Saúde, Departamento de Atenção Básica. Estratégias para o cuidado da pessoa com doença crônica: obesidade. Brasília: Ministério da Saúde, Cadernos de Atenção Básica. 2014; 212 p.: il. - n. 38.

2. Sociedade Brasileira de Cirurgia Bariátrica. Consenso Brasileiro Multissocietário em Cirurgia da Obesidade. São Paulo. 2006.

3. Abeso - Associação Brasileira para o Estudo da Obesidade e da Síndrome Metabólica. Diretrizes brasileiras de obesidade 2009/2010. Itapevi, SP : AC Farmacêutica, 2009; 3.ed.

4. Brasil. Ministério da Saúde, Secretaria de Vigilância em Saúde, Departamento de Vigilância de Doenças e Agravos não Transmissíveis e Promoção da Saúde. Vigitel Brasil 2015: vigilância de fatores de risco e proteção para doenças crônicas por inquérito telefônico - Brasília: Ministério da Saúde, 2016.

5. Prevedello CF, Colpo E, Mayer ET, Copetti H. Análise do impacto da cirurgia bariátrica em uma população do centro do estado do Rio Grande do Sul utilizando o método BAROS. Arq Gastroenterol. jul./set. 2009; v. 46 no.3.

6. Bordalo LA, Teixeira TFS, Bressan J, Mourão DM. Cirurgia bariátrica: como e por que suplementar. Rev. Assoc. Med. Bras. 2011; 57(1): 113-120.

7. Camberos-Solis $\mathrm{R}$, Jimenez-Cruz $\mathrm{A}$, Bacardí-Gascón M, Culebras JM. Efectividad y seguridad a largo plazo del bypass gástrico en " $Y$ " de Roux y de la banda gástrica: revisión sistemática. Nutr. Hosp. 2010; 25(6).

8. Karmali S, Brar B, Shi X, Sharma AM, de Gara C, Birch DW. Weight recidivism postbariatric surgery: a systematic review. Obes Surg. 2013; 23(11): 1922-33.

9. Khawali $C$, Ferraz $M B$, Zanella $M T$, Ferreira SRG. Evaluation of quality of life in severely obese patients after bariatric surgery carried out in the public healthcare system. Arq Bras Endocrinol Metab. 2012; 56(1): 33-38.

10. Monteiro FC, Silva WS, Filho NS, Ferreira PAM, Araújo GF, Mandarino NR, et al. Efeito da perda ponderal induzida pela cirurgia bariátrica sobre a prevalência de síndrome metabólica. Arq Bras Cardiol 2009; 92(6):452-456.

11. Ravelli MN, Merhi VAL, Mônaco DV, Aranha N. Obesidade, cirurgia bariátrica e implicações nutricionais. RBPS. 2007; 20 (4): 259266.

12. Silva PRB, Souza MR, Silva EM, Silva SA. Estado nutricional e qualidade de vida em pacientes submetidos à cirurgia bariátrica. $A B C D$ Arq Bras Cir Dig 2014;27(Suplemento 1):35-38.

13. Leiro LS, Melendez-Araújo MS. Adequação de micronutrientes da dieta de mulheres após um ano de bypass gástrico. $A B C D$ 
Arq Bras Cir Dig. 2014; 27 (Suplemento 1): 21-25. 14. Santos TD, Burgos MGPA, Lemos MCC, Cabral PC. Aspectos clínicos e nutricionais em mulheres obesas durante o primeiro ano após bypass gástrico em y-de-roux. ABCD Arq Bras Cir Dig 2015;28 (Supl.1):56-60.

15. Pech N, Meyer F, Lippert H, Manger T, Stroh C. Complications and nutrient deficiencies two years after sleeve gastrectomy. BMC Surgery. 2012; 12:13.

16. Carvalho IR, Loscalzo IT, Freitas MFB, Jordão RE, Friano TC. Incidência da deficiência de vitamina b12 em pacientes submetidos à cirurgia bariátrica pela técnica Fobicapella ( $y$-de-roux). ABCD Arq Bras Cir Dig. 2012; 25(1):36-40.

17. Dogan K, Aarts EO, Koehestanie P, Betzel $\mathrm{B}$, Ploeger $\mathrm{N}$, Boer $\mathrm{H}$, et al. Optimization of Vitamin Suppletion After Roux-En-Y GastricBypass Surgery Can Lower Postoperative Deficiencies. Medicine, Journal. November 2014; Volume 93, Number 25.

18. Ramos NMCPJ, Magno FCCM, Cohen L, Rosado EL, Carneiro JRI. Perda ponderal e presença de anemias carenciais em pacientes submetidos à bypass gástrico em y-de-roux em uso de suplementação de vitaminas e minerais. ABCD Arq Bras Cir Dig. 2015;28(1):44-47.

19. Costa LD, Valezi AC, Matsuo T, Dichi I, Dichi JB. Repercussão da perda de peso sobre parâmetros nutricionais e metabólicos de pacientes obesos graves após um ano de gastroplastia em Y-de-Roux. Rev. Col. Bras. Cir. 2010; 37(2): 096-101.

20. Silveira-Júnior $S$, Albuquerque $M M$, Nascimento RR, Rosa LS, Hygidio DA, Zapelini RM. Repercussões nutricionais em pacientes submetidos à cirurgia bariátrica. ABCD Arq Bras Cir Dig. 2015;28(1):48-52.

21. Eltweri AM, Bowrey DJ, Sutton CD, Graham L, Williams RN. An audit to determine if vitamin b12 supplementation is necessary after sleevegastrectomy. SpringerPlus Journal. 2013; 2:218.

22. Eussen SJ, de Groot LC, Joosten LW, Bloo RJ, Clarke R, Ueland PM, et al. Effect of oral vitamin $\mathrm{B} 12$ with or without folic acid on cognitive function in older people with mild vitamin B12 deficiency: A randomized placebocontrolled trial. Am J Clin Nutr. 2006; 84:361-70.

23. Tedesco AK, Biazotto R, Gebara TSS, Cambi MPC, Baretta GAP. Pré e pós-operatório de cirurgia bariátrica: algumas alterações bioquímicas. ABCD Arq Bras Cir Dig. 2016; 29(Supl.1):67-71.

24. Nascimento TM, Alves A Jr, Nunes MAP, Freitas TRP, Silva MAFS, Alves MRM. Comparação do perfil hepático no pré e pós-operatório na cirurgia bariátrica: rede particular vs pública. ABCD Arq Bras Cir Dig. 2015; 28(4):274-277.

25. Alves LFA, Gonçalves RM, Cordeiro GV, Lauria MW, Ramos AV. Beribéri pós bypass gástrico: uma complicação não tão rara. Relato de dois casos e revisão da literatura. Arq Bras Endocrinol Metab. Junho/2006; vol 50, no.

26. Stol A, Gugelmin G, Lampa VM Jr, Frigulha C, Selbachet RA. Complicações e óbitos nas operações para tratar a obesidade mórbida. Arq Bras Cir Dig. 2011; 24(4):282-284.

27. CFM - Conselho Federal de Medicina. Resolução no. 2131/2015. Publicada no D.O.U., 13 Jan. 2016, Seção I, p.66.

28. Pedrosa IV, Burgos MGPA, Souza NC, Morais CN. Aspectos nutricionais em obesos antes e após a cirurgia bariátrica. Rev. Col. Bras. Cir. 2009; 36(4): 316-322.

29. Aasheim ET, Bjo“rkman S, Søvik TT, Engstro“m M, Hanvold SE, Mala T, et al. Vitamin status after bariatric surgery: a randomized study of gastric bypass and duodenal switch. Am J Clin Nutr. 2010; 90:15-22.

30. Xanthakos SA. Nutritional Deficiencies in Obesity and After Bariatric Surgery. Pediatr Clin North Am. Outubro/2009; 56(5): 1105-1121.

31. Wrzesinski A, Corrêa JM, Fernandes TMB, Monteiro LF, Trevisol FS, Nascimento RR. Complicações que necessitaram de manejo hospitalar no pós-operatório de cirurgia bariátrica. ABCD Arq Bras Cir Dig. 2015;28(Supl.1):3-6.

32. Rocha JCG. Deficiência de vitamina B12 no pós-operatório de cirurgia bariátrica. International Journal of Nutrology. Maio/agosto 2012; v.5, n.2, p. 82-89.

33. Rubio MA, Moreno C. Implicaciones nutricionales de la cirugia bariatrica sobre el tracto gastrointestinal. Nutr Hosp. Maio/2007; 22 Suppl 2:124-34.

34. Menegotto ALS, Cruz MRR, Soares FL, Nunes MGJ, Branco-Filho AJ. Avaliação da frequência em consultas nutricionais dos pacientes após cirurgia bariátrica. $A B C D$ Arq Bras Cir Dig. 2013; (2):117-119.

35. Fábregas BC, Vitorino FD, Teixeira A.L. Deficiencia de vitamina B12 e transtorno depressivo refratario. Relato de caso. J Bras Psiquiatr. 2011; 60(2):141-143.

36. Schweiger C, Weiss R, Keidar A. Effect of different bariatric operations on food tolerance and quality of eating. Obes Surg. Outubro/2010; 20(10):1393-9.

37. Traina F. Deficiência de ferro no paciente submetido à ressecção gástrica ou intestinal: prevalência, causas, repercussões clínicas, abordagem diagnóstica e prevenção. 
Rev. Bras. Hematol. Hemoter. 2010; 32(Supl. 2):78-83.

38. Moreira MA, Espínola PRM, Azevedo CW. Intolerâncias alimentares e sintomas associados em pacientes submetidos à técnica de Fobi-Capella sem anel gástrico. ABCD Arq Bras Cir Dig. 2015; 28(1):36-39.

39. Cambi MPC, Marchesini SD, Baretta GAP. Reganho de peso após cirurgia bariátrica: avaliação do perfil nutricional dos pacientes candidatos ao procedimento de plasma endoscópico de argônio. $A B C D$ Arq Bras Cir Dig. 2015; 28(1):40-43.

40. Shankar P, Boylan M, Sriram K. Micronutrient deficiencies after bariatric surgery. Nutrition. 2010; 26(11-12):1031-7.

\section{Endereço para Correspondência}

Centro Universitário de Saúde, Ciências Humanas e Tecnológicas do Piauí - UNINOVAFAPI

Rua Vitórino Orthiges Fernandes, 6123 - Uruguai, Teresina - PI

CEP.: 64073-505

e-mail: dourado_ariana@hotmail.com

Recebido em 24/07/2017

Aprovado em 05/02/2018

Publicado em 30/03/2018 